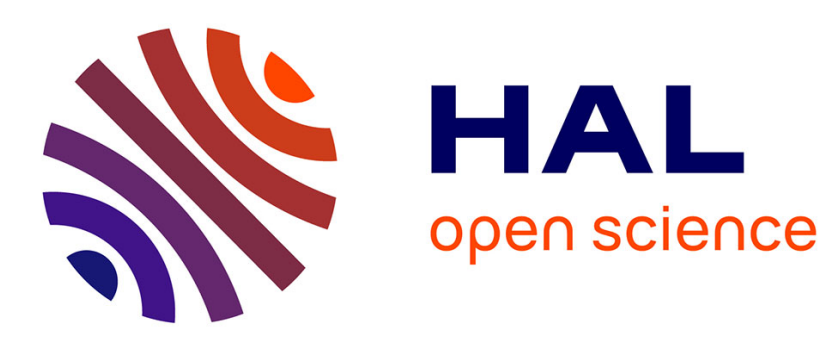

\title{
Integral expressions of the small-angle scattering correlation-function derivatives
}

S. Ciccariello

\section{To cite this version:}

S. Ciccariello. Integral expressions of the small-angle scattering correlation-function derivatives. Journal de Physique IV Proceedings, 1993, 03 (C8), pp.C8-499-C8-502. 10.1051/jp4:19938103 . jpa00252233

\section{HAL Id: jpa-00252233 https://hal.science/jpa-00252233}

Submitted on 1 Jan 1993

HAL is a multi-disciplinary open access archive for the deposit and dissemination of scientific research documents, whether they are published or not. The documents may come from teaching and research institutions in France or abroad, or from public or private research centers.
L'archive ouverte pluridisciplinaire HAL, est destinée au dépôt et à la diffusion de documents scientifiques de niveau recherche, publiés ou non, émanant des établissements d'enseignement et de recherche français ou étrangers, des laboratoires publics ou privés. 


\title{
Integral expressions of the small-angle scattering correlation-function derivatives
}

\section{S. CICCARIELLO}

Dipartimento di Fisica 'G. Galilei' and sez. I.N.F.M., via Marzolo 8, 35131 Padova, Italy

\begin{abstract}
The integral expression of the nth derivative of the SAS correlationfunction $(\gamma(r))$ is reported. It is the average, throughout the sample interface, of the loop integral of a vectorial field $\overrightarrow{\mathcal{A}}^{(n)}\left(\mathbf{r}_{j}(l)\right)$. All the $\overrightarrow{\mathcal{A}}^{(n)}\left(\mathbf{r}_{j}(l)\right)$ 's, with $n \geq \mathbf{3}$, are recursively obtained by appropriate differentiation of $\overrightarrow{\mathcal{A}}^{(2)}\left(\mathbf{r}_{j}(l)\right)$, whose explicit expression is given in terms of the parametric equations of the interface. For very smooth interface it results that all the $\mathrm{CF}$ even-derivatives are null at the origin.
\end{abstract}

1. The small-angle intensity $(\equiv I(h))$ scattered from an isotropic system, which can be modelled as a set of homogeneous regions $V_{i}$, fully characterized by the electron or scattering-length densities $n_{i}(i=1, . . N)$, is the $3-D$ Fourier transform of the so-called correlation function (CF)

$$
\gamma(r)=1-\sum_{i, j=1}^{N} \frac{\left(n_{i}-n_{j}\right)^{2}}{\left\langle\eta^{2}\right\rangle} P_{i j}(r)
$$

Here $\left\langle\eta^{2}>\right.$ denotes the mean square density fluctuation, while $P_{i j}(r)$ is the stick probability function (SPF) relevant to the ith and jth phase. The SPF's depend only on the geometrical configurations of the involved phases since their definition is

$$
P_{i j}(r)=(4 \pi V)^{-1} \int_{\underline{\underline{r}}_{i}} d v_{i} \int_{\underline{\underline{r}}_{j}} d v_{j} \int_{\mathcal{B}^{2}} d \hat{\omega} \delta\left(\mathbf{r}_{i}+r \hat{\omega}-\mathbf{r}_{j}\right) .
$$

Investigating how the geometrical features of the boundaries of sets $V_{i}$ reflect into the behaviour of the $P_{i j}$ 's and of their derivatives is theoretically interesting and the results $[1,2,3]$ can be of some practical relevance for the noticed relation between $I(h)$ and $\gamma(r)$. Our aim here, is to report the general integral expression of the nth $\gamma(r)$ derivative in terms of the parametric equations of the phase boundaries. 
2. By applying twice the mathematical identity

$$
\begin{aligned}
\frac{d}{d r} \delta\left(\mathbf{r}_{i}+r \hat{\omega}-\mathbf{r}_{j}\right) & =\hat{\omega} \cdot \nabla \delta\left(\mathbf{r}_{i}+\mathbf{r}-\mathbf{r}_{j}\right)= \\
\nabla_{i} \cdot\left(\hat{\omega} \delta\left(\mathbf{r}_{i}+r \hat{\omega}-\mathbf{r}_{j}\right)\right) & =-\nabla_{j} \cdot\left(\hat{\omega} \delta\left(\mathbf{r}_{i}+r \hat{\omega}-\mathbf{r}_{j}\right)\right)
\end{aligned}
$$

to eq. (2) and by Gauss' theorem it results[4]

$$
4 \pi V r^{2} P_{i j}^{\prime \prime}(r)=-\int_{\underline{S}_{i}} d S_{i} \int_{\underline{S}_{j}} d S_{j} \int_{\underline{S}_{\left(\mathbf{r}_{i}, r\right)}} d S\left(\hat{\sigma}_{i} \cdot \hat{\omega}\right)\left(\hat{\sigma}_{j} \cdot \hat{\omega}\right) \delta\left(\mathbf{r}_{i}+r \hat{\omega}-\mathbf{r}_{j}\right),
$$

with $\hat{\sigma}_{i}$ denoting the unit vector orthogonal to $d S_{i}$. The Dirac function requires that the point $\mathbf{r}_{i}+r \hat{\omega}$ of $\underline{S}\left(\mathbf{r}_{i}, r\right)$ (the boundary of the sphere of radius $r$ centered at $\mathbf{r}_{i}$ ) be equal to $\mathbf{r}_{j}$, a point lying on $\underline{S}_{j}$. Therefore the two inner integrals on the right hand side (rhs) of (4) amount to a curvilinear integral performed along the curve $\underline{\Gamma}_{j}\left(\mathbf{r}_{i}, r\right)$ resulting from the intersection of $\underline{S}\left(\mathbf{r}_{i}, r\right)$ with $\underline{S}_{j}$. It follows that

$$
P_{i j}^{\prime \prime}(r)=-\frac{1}{4 \pi V} \int_{\underline{S}_{i}} d S_{i} p_{i j}^{(2)}\left(\mathbf{r}_{i}, r\right)
$$

with

$$
p_{i j}^{(2)}\left(\mathbf{r}_{i}, r\right)=\oint_{\underline{\Gamma}_{j}\left(\mathbf{r}_{i}, r\right)} \overrightarrow{d l} \cdot \overrightarrow{\mathcal{A}}^{(2)}\left(\mathbf{r}_{j}(l)\right)
$$

i.e. $p_{i j}^{(2)}\left(\mathbf{r}_{i}, r\right)$ is the loop integral along $\Gamma_{j}\left(\mathbf{r}_{i}, r\right)$ of the vectorial field

$$
\overrightarrow{\mathcal{A}}^{(2)}\left(\mathbf{r}_{j}(l)\right) \equiv \frac{\left[\hat{\sigma}_{i} \cdot \hat{\omega}\left(\mathbf{r}_{j}(l)\right)\right]\left[\hat{\sigma}_{j}\left(\mathbf{r}_{j}(l)\right) \cdot \hat{\omega}\left(\mathbf{r}_{j}(l)\right)\right]}{\left\|\mathbf{r}_{j}(l)-\mathbf{r}_{i}\right\|^{2} \sqrt{1-\left[\hat{\sigma}_{j}\left(\mathbf{r}_{j}(l)\right) \cdot \hat{\omega}\left(\mathbf{r}_{j}(l)\right)\right]^{2}}} \hat{\tau}_{j}\left(\mathbf{r}_{j}(l)\right) .
$$

This depends on $\hat{\sigma}_{j}$ and on $\hat{\tau}_{j}\left(\mathbf{r}_{j}(l)\right)$ (the unit vector tangent to $\underline{\Gamma}_{j}\left(\mathbf{r}_{i}, r\right)$ at the point $\mathbf{r}_{j}(l)$ ) and it is fully determined once the parametric equations of $\underline{S}_{j}$ are known. The further derivation of (4) yields

$$
(4 \pi V) P_{i j}^{(3)}(r)=-\int_{\mathcal{B}^{2}} d \hat{\omega} \int_{\underline{S}_{i}} d S_{i} \int_{\underline{S}_{j}} d S_{j}\left(\hat{\sigma}_{i} \cdot \hat{\omega}\right)\left(\hat{\sigma}_{j} \cdot \hat{\omega}\right)\left(\hat{\omega} \cdot \nabla_{i}\right) \delta\left(\mathbf{r}_{i}+r \hat{\omega}-\mathbf{r}_{j}\right)
$$

Again, by the same argument used above, the two inner integral amount to a curvilinear integral along $\underline{\Gamma}_{j}\left(\mathbf{r}_{i}, r\right)$. It has recently been shown that [5]

$$
P_{i j}^{(3)}(r)=-\frac{1}{4 \pi V} \int_{\underline{S}_{i}} d S_{i} p_{i j}^{(3)}\left(\mathbf{r}_{i}, r\right)
$$

with

$$
p_{i j}^{(3)}\left(\mathbf{r}_{i}, r\right) \equiv \int_{\underline{\Gamma}_{i}\left(\mathbf{r}_{i}, r\right)} \overrightarrow{d l} \cdot \overrightarrow{\mathcal{A}}^{(3)}\left(\mathbf{r}_{j}(l)\right) \quad \forall n \geq 2
$$


and

$$
\overrightarrow{\mathcal{A}}^{(3)}\left(\mathbf{r}_{j}\right) \equiv \frac{\mathbf{r}_{j, u} \cdot \overrightarrow{\mathcal{A}}_{, v}^{(2)}\left(\mathbf{r}_{j}\right)-\mathbf{r}_{j, v} \cdot \overrightarrow{\mathcal{A}}_{, u}^{(2)}\left(\mathbf{r}_{j}\right)}{\left\|\mathbf{r}_{j, u} \times \mathbf{r}_{j, v}\right\| \sqrt{1-\left(\hat{\sigma}_{j} \cdot \hat{\omega}\right)^{2}}} \tau_{j}\left(\mathbf{r}_{j}\right)
$$

Recalling that the knowledge of the parametric equations of $\underline{S}_{j}$ amounts to knowing $\mathbf{r}_{j}=$ $\mathbf{r}_{j}(u, v)$ and since $\overrightarrow{\mathcal{A}}_{, v}^{(2)}\left(\mathbf{r}_{j}\right)$ denotes the partial $v$-derivative of $\overrightarrow{\mathcal{A}}^{(2)}$, it is clear that the vectorial field $\overrightarrow{\mathcal{A}}^{(3)}$, defined on the variety $\underline{S}_{j}$, is fully determined. Thus, eq.s (8) represent the sought for integral expression of $P_{i j}^{(3)}(r)$. Provided the relevant derivatives exist, the analysis can be extended to higher order derivatives and one ends up with the following 'iterative' structure

$$
P_{i j}^{(n)}(r)=-\frac{1}{4 \pi V} \int_{\underline{S}_{i}} d S_{i} p_{i j}^{(n)}\left(\mathbf{r}_{i}, r\right) \quad \forall n \geq 2
$$

with

$$
p_{i j}^{(n)}\left(\mathbf{r}_{i}, r\right) \equiv \int_{\underline{\Gamma}_{j}\left(\mathbf{r}_{i}, r\right)} \overrightarrow{d l} \cdot \overrightarrow{\mathcal{A}}^{(n)}\left(\mathbf{r}_{j}(l)\right) \quad \forall n \geq 2
$$

and

$$
\vec{A}^{(n)}\left(\mathbf{r}_{j}\right) \equiv \frac{\mathbf{r}_{j, u} \cdot \overrightarrow{\mathcal{A}}_{, v}^{(n-1)}\left(\mathbf{r}_{j}\right)-\mathbf{r}_{j, v} \cdot \overrightarrow{\mathcal{A}}_{, u}^{(n-1)}\left(\mathbf{r}_{j}\right)}{\left\|\mathbf{r}_{j, u} \times \mathbf{r}_{j, v}\right\| \sqrt{1-\left(\hat{\sigma}_{j} \cdot \hat{\omega}\right)^{2}}} \hat{\tau}_{j}\left(\mathbf{r}_{j}\right), \quad \forall n \geq 3
$$

3. By these expressions it is possible to analyze the behaviour of the $P_{i j}^{(n)}(r)$ 's as $r \rightarrow 0$. It is recalled that these values determine the $\gamma^{(n)}(0)$-values and, consequently, the coefficients of the asymptotic expansion of $I(h)$ at large $h$ 's. Being $\overrightarrow{\mathcal{A}}^{(n)}\left(\mathbf{r}_{j}\right)$ parallel to $\hat{\tau}_{j}\left(\mathbf{r}_{j}\right)$, the following decomposition holds true

$$
\overrightarrow{\mathcal{A}}^{(n)}\left(\mathbf{r}_{j}\right)=a^{(n)}(r, \varphi) \hat{\omega}_{, \varphi} / \sin \theta+b^{(n)}(r, \varphi) \hat{\omega}_{, \theta} \quad \forall n \geq 2
$$

once the polar-coordinate parameterizations is used. It results

$$
p_{i j}^{(n)}\left(\mathbf{r}_{i}, r\right)=\int_{0}^{2 \pi} d \varphi A^{(n)}(r, \varphi) \quad \forall n \geq 3
$$

with $A^{(n)}$ simply related to $a^{(n)}$. When $n(>1)$ is even, the limits of the $A^{(n)}(r, \varphi)$ 's, as $r \rightarrow 0$, are antiperiodic functions, in the sense that $A^{(n)}(0, \varphi+\pi)=-A^{(n)}(0, \varphi)$. Thus, from eq. (11) and $(9 \mathrm{a})$ it follows that all the even derivatives of $\gamma(r)$ are null at $r=0$, provided the phase boundaries are sufficiently regular for the r-expansion of $A^{(n)}(r, \varphi)$ to exist. It should be noted that this property had been conjectured by Wu and Schmidt in $[3 b]$ on the basis of their findings that $\gamma^{(2)}\left(0^{+}\right)=\gamma^{(4)}\left(0^{+}\right)=0$. 
The explicit evaluation of the odd derivatives at $r=0$ can be carried through by the same technique. The third derivative yields the Kirste-Porod[2] result. The expression of the fifth derivative is

$$
P_{i j}^{(5)}\left(0^{+}\right)=-\frac{S_{i j}}{4 V}\left\langle\left[24 \mathcal{J}_{1}+12 \mathcal{J}_{2}-24 \mathcal{J}_{3}\right]\right\rangle_{i j}
$$

where $\left\langle\cdot>_{i j}\right.$ denotes the average over the $i j$-interface of quantities $\mathcal{J}_{l}$, defined as:

$$
\begin{gathered}
\mathcal{J}_{1} \equiv\left[5 q_{2,0} q_{4,0}+q_{2,0} q_{4,2}+q_{2,0} q_{4,4}+q_{2,2} q_{4,0}+q_{2,2} q_{4,2}+5 q_{2,2} q_{4,4}\right] / 8 \\
\mathcal{J}_{2} \equiv\left[5 q_{3,0}^{2}+2 q_{3,0} q_{3,2}+2 q_{3,1} q_{3,3}+q_{3,1}^{2}+q_{3,2}^{2}+5 q_{3,3}^{2}\right] / 8
\end{gathered}
$$

and

$$
\mathcal{J}_{3} \equiv\left[35\left(q_{2,0}^{4}+q_{2,2}^{4}\right)+20 q_{2,0} q_{2,2}\left(q_{2,0}^{2}+q_{2,2}^{2}\right)+18\left(q_{2,0} q_{2,2}\right)^{2}\right] / 64
$$

with the $q_{m, n}$ 's equal to the coefficients of the power-series expansion of the interface equation with respect to a cartesian system having the $X-Y$ plane tangent to the surface and the $Z$-axis directed along the normal at the tangency point set at the origin, i.e.

$$
Z=\sum_{n=2} \sum_{m=0}^{n} q_{n, m} X^{n-m} Y^{m}
$$

For spherical interfaces, the $\mathcal{J}_{l}$ 's are easily checked to be null. Finally, it is noted that result (12) was obtained by $\mathrm{Wu}$ and Schmidt $[3 b]$ ( see eq. (13) and the three definitions immediately below) under the more restrictive assumption that the sample is a dilute two-phase system of convex particles.

\section{References}

/1/ Méring J. and Tchoubar D., J. Appl. Cryst. 1 (1968) 153; Tchoubar D. and Méring J., J. Appl. Cryst. 2 (1969) 128.

/2/ Kirste R. and Porod G., Kolloid-Zeit 184 (1962) 1.

/3/ a)Schmidt P.W., J. Math. Phys.(NY) 6 (1965) 424; b) Wu H. and Schmidt P.W., J. Appl. Cryst. 4 (1971) 224; c) Wu H. and Schmidt P.W., J. Appl. Cryst. 7 (1974) 131.

/4/ Ciccariello S., Cocco G., Benedetti A. and Enzo S., Phys. Rev. B23 (1981) 6474.

/5/ Ciccariello S., preprint DFPD 92/Th/44. 